\title{
Computing Some Degree-Based Topological Indices of Graphene
}

\author{
Vijayalaxmi Shigehalli, Rachanna Kanabur* \\ Deparment of Mathematics \\ Rani Channamma University, Belagavi-591156 \\ Karnataka, India \\ e-mail: rachukanabur@gmail.com
}

\begin{abstract}
Graphene is one of the most promising nanomaterial because of its unique combination of superb properties, which opens a way for its exploitation in a wide spectrum of applications ranging from electronics to optics, sensors, and bio devices. Inspired by recent work on Graphene of computing topological indices, here we compute new topological indices viz. Arithmetic-Geometric index (AG2 index), SK3 index and Sanskruti index of a molecular graph $G$ and obtain the explicit formulae of these indices for Graphene.
\end{abstract}

Keywords: Arithmetic-Geometric index (AG2 index), SK3 index, sanskruti index, graphene.

\section{Introduction}

A molecular graph is a representation of a chemical compound having atoms as vertices and the bonds between atoms correspond to the edge of the graph. The collection of vertices of a graph, says $G$, is denoted by $V(G)$ and the set of edges is denoted by $E(G)$. The degree of a vertex $v$ of a graph is the number of vertices of $G$ adjacent to $v$, denoted by $d G(v)$ or simply as dv [3].

Molecular graphs are a special type of chemical graphs, which represent the constitution of molecules. They are also called constitutional graphs. When the constitutional graph of a molecule is represented in a two-dimentional basis it is called structural graphs $[7,8]$.

Topological invariant of a graph is a single number descriptor which is correlated to certain chemical, thermodynamical and biological behavior of the chemical compounds. Several topological indices have been defined over past decades which depend on degree of vertices.

\section{Computing the Topological Indices of Graphene.}

Graphene is an atomic scale honeycomb lattice made of carbon atoms. Graphene is 200 times stronger than steel, one million times thinner than a human hair, and world's most conductive material. So it has captured the attention of scientists, researchers, and industries worldwide. It is one of the most promising nanomaterials because of its unique combination of superb properties, which opens a way for its exploitation in a wide spectrum of applications ranging from electronics to optics, sensors, and biodevices. Also it is the most effective material for electromagnetic interference (EMI) shielding. Now we focus on computation of topological indices of Graphene [1, 4].

Motivated by previous research on Graphene [4,6,11-14], here we compute three new topological indices viz. Arithmetic-Geometric index (AG2 index), SK3 index and Sanskruti index of a molecular graph $\mathrm{G}$ and obtain the explicit formulae of these indices for Graphene.

\subsection{Arithmetic-Geometric $\left(A G_{2}\right)$ Index}

Let $G=(V, E)$ be a molecular graph, and $S G(u)$ is the degree of the vertex $u$, then AG2 index of $G$ is defined as

$$
\operatorname{AG2}(\mathrm{G})=\sum_{u, v \in E(G)} \frac{S_{G}(u)+S_{G}(v)}{2 \sqrt{S_{G}(u) \cdot S_{G}(v)}}
$$


where, AG2 index is considered for distinct vertices.

The above equation is the sum of the ratio of the Arithmetic mean and Geometric mean of $u$ and $v$, where $S G(u)$ (or $S G(v)$ ) is the summation of degrees of all neighbours of vertex $u$ (or v) in $\mathrm{G}$.

$$
\sum_{\mathrm{SG}(\mathrm{u})=u, v \in E(G)} d_{G}(u) \text { and NG }(\mathrm{u})=\{\mathrm{v} \in \mathrm{V}(\mathrm{G}) / \mathrm{uv} \in \mathrm{E}(\mathrm{G})\}
$$

\section{2. $S K_{3}$ Index}

The SK3 index of a graph $G=(V, E)$ is defined as

$$
\operatorname{SK3~}(\mathrm{G})=\sum_{u, v \in E(G)} \frac{S_{G}(u)+S_{G}(v)}{2}
$$

where $S G(u)$ (or $S G(v)$ ) is the summation of degrees of all neighbours of vertex $u($ or $v$ ) in $G$.

$$
\sum_{\mathrm{SG}(\mathrm{u})=u, v \in E(G)} d_{G}(u) \text { and } \mathrm{NG}(\mathrm{u})=\{\mathrm{v} \in \mathrm{V}(\mathrm{G}) / \mathrm{uv} \in \mathrm{E}(\mathrm{G})\}
$$

\subsection{Sanskruti Index}

Recently, Hosamani [9], studied a novel topological index, namely the Sanskruti index S (G) of a molecular graph $G$, which is denoted as

$$
S(G)=\sum_{u v \in E(G)}\left(\frac{S_{G}(u) \cdot S_{G}(v)}{S_{G}(u)+S_{G}(v)-2}\right)^{3}
$$

where $S G(u)$ (or $S G(v))$ is the summation of degrees of all neighbours of vertex $u($ or $v$ ) in $G$.

$$
\mathrm{SG}(\mathrm{u})=\sum_{u, v \in E(G)} d_{G}(u) \text { and NG }(\mathrm{u})=\{\mathrm{v} \in \mathrm{V}(\mathrm{G}) / \mathrm{uv} \in \mathrm{E}(\mathrm{G})\}
$$

\section{Main Results}

\subsection{The $A G_{2}$ index of Graphene is}

$$
A G_{2}(G)=\left\{\begin{array}{lc}
6.0588 t+2.0277 s+3 t s-1.0032 & \text { if } t \neq 1 \\
5.0119 s+1.0578 & \text { if } t=1 \\
6 & \text { if } t=1, s>1
\end{array}\right.
$$

Let $e_{i, j}$ denote the number of edges with $i=S u$ and $j=S v$. It is easy to see that the summation of degrees of edge endpoints of Graphene has nine edge types $e_{4,5}, e_{5,5}, e_{5,7}, e_{5,8}, e_{6,7}$, $e_{7,9}, e_{8,8}, e_{8,9}$ and $e_{9,9}$ that are enumerated Table 1. For convenience these edge type are colored by grey, yellow, red, purple, blue, green, light blue, brown, and black, respectively, as shown in Figure 1. 

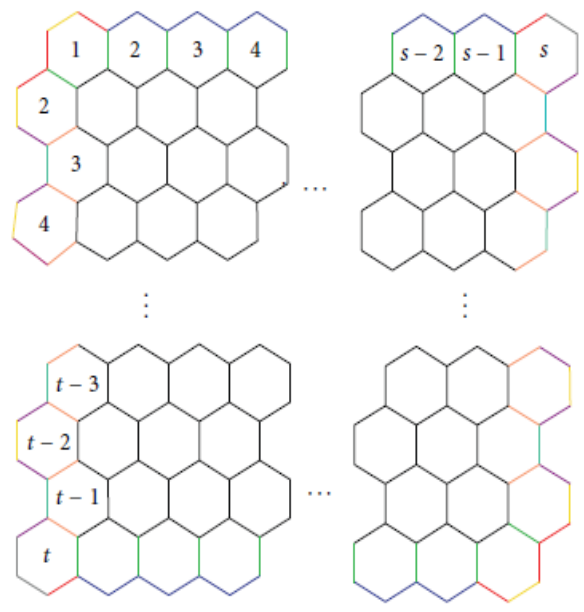

Figure 1. Colors of Graphene Edge (1)

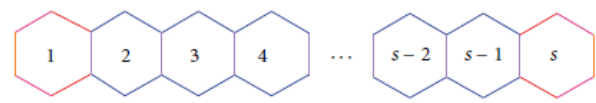

Figure 2. Colors of Graphene Edge (2)

Case 1. The $A G_{2}$ index of Graphene for $t \neq 1$ is

$$
A G_{2}(G)=\sum_{u, v \in E(G)} \frac{S_{G}(u)+S_{G}(v)}{2 \sqrt{S_{G}(u) \cdot S_{G}(v)}}
$$

Table 1. Nine edge types of Graphene

\begin{tabular}{|c|c|c|c|c|c|c|c|c|c|}
\hline Rows & $e_{4,5}$ & $e_{5,5}$ & $e_{5,7}$ & $\mathrm{e}_{5,8}$ & $e_{6,7}$ & $e_{7,9}$ & $e_{8,8}$ & $e_{8,9}$ & $e_{9,9}$ \\
\hline 1 & 2 & 1 & 3 & 1 & $2 s-4$ & $\mathrm{~s}$ & 0 & 1 & $2 s-3$ \\
\hline 2 & 0 & 1 & 1 & 1 & 0 & 0 & 1 & 2 & $3 s-4$ \\
\hline 3 & 0 & 1 & 0 & 2 & 0 & 0 & 1 & 2 & $3 s-4$ \\
\hline 4 & 0 & 1 & 0 & 2 & 0 & 0 & 1 & 2 & $3 s-4$ \\
\hline . & . & . & . & . & . & . & . & . & . \\
\hline . & . & . & . & . & . & . & . & . & . \\
\hline$t-2$ & 0 & 1 & 0 & 2 & 0 & 0 & 1 & 2 & $3 s-4$ \\
\hline$t-1$ & 0 & 1 & 1 & 1 & 0 & 0 & 1 & 1 & $3 s-4$ \\
\hline$t$ & 2 & 1 & 3 & 1 & $2 s-4$ & $\mathrm{~s}$ & 0 & 0 & 0 \\
\hline Total & 4 & $t$ & 8 & $2 \mathrm{t}-4$ & $4 s-8$ & $2 s$ & $t-2$ & $2 \mathrm{t}-4$ & $\begin{array}{l}3 t s-4 s- \\
4 t+5\end{array}$ \\
\hline
\end{tabular}

$$
\begin{aligned}
\mathrm{AG}_{2}(\mathrm{G})= & \left(e_{4,5}\right)\left(\frac{4+5}{2 \sqrt{20}}\right)+\left(e_{5,5}\right)\left(\frac{5+5}{2 \sqrt{25}}\right)+\left(e_{5,7}\right)\left(\frac{5+7}{2 \sqrt{35}}\right)+\left(e_{5,8}\right)\left(\frac{5+8}{2 \sqrt{40}}\right)+\left(e_{6,7}\right) \\
& \left(\frac{6+7}{2 \sqrt{42}}\right)\left(e_{7,9}\right)\left(\frac{7+9}{2 \sqrt{63}}\right)+\left(e_{8,8}\right)\left(\frac{8+8}{2 \sqrt{64}}\right)+\left(e_{9,8}\right)\left(\frac{9+8}{2 \sqrt{72}}\right)+\left(e_{9,9}\right)\left(\frac{9+9}{2 \sqrt{81}}\right) \\
= & (4)\left(\frac{9}{2 \sqrt{20}}\right)+(t)\left(\frac{10}{2 \sqrt{25}}\right)+(8)\left(\frac{12}{2 \sqrt{35}}\right)+(2 t-4)\left(\frac{13}{2 \sqrt{40}}\right)+(4 s-8)\left(\frac{13}{2 \sqrt{42}}\right)
\end{aligned}
$$




$$
\begin{aligned}
+ & (2 s)\left(\frac{16}{2 \sqrt{63}}\right)+(t-2)\left(\frac{16}{2 \sqrt{64}}\right)+(2 t-4)\left(\frac{17}{2 \sqrt{72}}\right)+(3 t s-4 s-4 t+5)\left(\frac{18}{2 \sqrt{81}}\right) \\
= & \left(5+\frac{13}{\sqrt{40}}+1+\frac{17}{\sqrt{72}}-4\right) t+\left(\frac{26}{\sqrt{42}}+\frac{16}{\sqrt{63}}-4\right) s+3 t s+ \\
& \left(\frac{18}{\sqrt{20}}+\frac{48}{\sqrt{35}}-\frac{26}{\sqrt{40}}-\frac{52}{\sqrt{42}}-2-\frac{34}{\sqrt{72}}+5\right) \\
= & 6.0588 \mathrm{t}+2.0277 \mathrm{~s}+3 \mathrm{ts}-1.0032
\end{aligned}
$$

Case 2. For $t=1$ and $s>1$, Graphene has five types of edges, namely $e_{4,4}, e_{4,5}, e_{5,7}, e_{6,7}$, and $e_{7,7}$. These edges are colored in orange, pink, red, blue, and lavender, respectively, as shown in Figure 2. The number of edges of these types is shown in Table 2

Table 2. The Number of Edges

\begin{tabular}{cccccc}
\hline $\begin{array}{c}\text { Number of } \\
\text { benzene rings(s) }\end{array}$ & $\mathrm{e}_{4,4}$ & $\mathrm{e}_{4,5}$ & $\mathrm{e}_{5,7}$ & $\mathrm{e}_{6,7}$ & $\mathrm{e}_{7,7}$ \\
\hline 2 & 2 & 4 & 4 & 0 & 1 \\
3 & 2 & 4 & 4 & 4 & 2 \\
4 & 2 & 4 & 4 & 8 & 3 \\
5 & 2 & 4 & 4 & 12 & 4 \\
. &. &. &. &. &. \\
. &. &. &. &. &. \\
s-1 & 2 & 4 & 4 & $4 \mathrm{~s}-12$ & $\mathrm{~s}-2$ \\
$\mathrm{~s}$ & 2 & 4 & 4 & $4 \mathrm{~s}-8$ & $\mathrm{~s}-1$ \\
\hline
\end{tabular}

$$
\begin{aligned}
\mathrm{AG}_{2}(\mathrm{G}) & =\left(e_{4,4}\right)\left(\frac{4+4}{2 \sqrt{16}}\right)+\left(e_{4,5}\right)\left(\frac{4+5}{2 \sqrt{20}}\right)+\left(e_{5,7}\right)\left(\frac{5+7}{2 \sqrt{35}}\right)+\left(e_{6,7}\right)\left(\frac{6+7}{2 \sqrt{42}}\right)+\left(e_{7,7}\right)\left(\frac{7+7}{2 \sqrt{49}}\right) \\
& =(2)\left(\frac{8}{2 \sqrt{16}}\right)+(4)\left(\frac{9}{2 \sqrt{20}}\right)+(4)\left(\frac{12}{2 \sqrt{35}}\right)+(4 s-8)\left(\frac{13}{2 \sqrt{40}}\right)+(s-1)\left(\frac{14}{2 \sqrt{49}}\right) \\
& =\left(2+\frac{18}{\sqrt{20}}+\frac{24}{\sqrt{35}}-\frac{52}{\sqrt{42}}-1\right) t+\left(\frac{26}{\sqrt{42}}+1\right) s \\
& =5.0119 \mathrm{~s}+1.0578
\end{aligned}
$$

Case 3 . For $t=1$ and $s=1$, we have only 6 edges of the type e 4,4 as shown in Figure 3 .

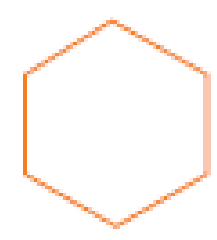

Figure 3. Type $e_{4,4}$ with 6 Edges 


$$
A G_{2}(G)=\left(e_{4,4}\right)\left(\frac{4+4}{2 \sqrt{16}}\right)=6
$$

\subsection{The $S K_{3}$ index of Graphene is}

$$
S K_{3}(G)=\left\{\begin{array}{lc}
7 t+6 s+27 t s-17 & \text { if } t \neq 1 \\
33 s-9 & \text { if } t=1 \\
24 & \text { if } t=1, s>1
\end{array}\right.
$$

Proof: Let $e_{i, j}$ denote the number of edges with $i=S u$ and $j=S v$. It is easy to see that the summation of degrees of edge endpoints of Graphene has nine edge types $e_{4,5}, e_{5,5}, e_{5,7}, e_{5,8}, e_{6,7}$, $e_{7,9,} e_{8,8}, e_{8,9}$ and $e_{9,9}$ that are enumerated Table1. For convenience these edge type are colored by grey, yellow, red, purple, blue, green, light blue, brown, and black, respectively, as shown in Figure1.

Case 1. The $S K_{3}$ index of Graphene for $t \neq 1$ is

$$
\begin{aligned}
& S K_{3}(G)=\sum_{u, v \in E(G)} \frac{S_{G}(u)+S_{G}(v)}{2} \\
& S K_{3}(G)=\left(e_{4,5}\right)\left(\frac{4+5}{2}\right)+\left(e_{5,5}\right)\left(\frac{5+5}{2}\right)+\left(e_{5,7}\right)\left(\frac{5+7}{2}\right)+\left(e_{5,8}\right)\left(\frac{5+8}{2}\right)+\left(e_{6,7}\right)\left(\frac{6+7}{2}\right)+ \\
&\left(e_{7,9}\right)\left(\frac{7+9}{2}\right)+\left(e_{8,8}\right)\left(\frac{8+8}{2}\right)+\left(e_{9,8}\right)\left(\frac{9+8}{2}\right)+\left(e_{9,9}\right)\left(\frac{9+9}{2}\right) \\
&=(4)\left(\frac{9}{2}\right)+(t)\left(\frac{10}{2}\right)+(8)\left(\frac{12}{2}\right)+(2 t-4)\left(\frac{13}{2}\right)+(4 s-8)\left(\frac{13}{2}\right)+(2 s)\left(\frac{16}{2}\right)+(t-2) \\
&\left(\frac{16}{2}\right)+(2 t-4)\left(\frac{17}{2}\right)+(3 t s-4 s-4 t+5)\left(\frac{18}{2}\right) \\
&=(5+13+8+17-36) t+(26+16-36) s+27 t s+(18+48-26-52-16-34+45) \\
&= 7 \mathrm{t}+6 \mathrm{~s}+27 \mathrm{ts}-17
\end{aligned}
$$

Case 2. For $t=1$ and $s>1$, Graphene has five types of edges, namely $e_{4,4}, e_{4,5}, e_{5,7}, e_{6,7}$, and $e_{7,7}$. These edges are colored in orange, pink, red, blue, and lavender, respectively, as shown in Figure 2. The number of edges of these types is shown in Table 2.

$$
\begin{aligned}
S K_{3}(G) & =\left(e_{4,4}\right)\left(\frac{4+4}{2}\right)+\left(e_{4,5}\right)\left(\frac{4+5}{2}\right)+\left(e_{5,7}\right)\left(\frac{5+7}{2}\right)+\left(e_{6,7}\right)\left(\frac{6+7}{2}\right)+\left(e_{7,7}\right)\left(\frac{7+7}{2}\right) \\
& =(2)\left(\frac{8}{2}\right)+(4)\left(\frac{9}{2}\right)+(4)\left(\frac{12}{2}\right)+(4 s-8)\left(\frac{13}{2}\right)+(s-1)\left(\frac{14}{2}\right) \\
& =(8+18+24-52-7) t+(26+7) s \\
& =33 s-9
\end{aligned}
$$


Case 3. For $t=1$ and $s=1$, we have only 6 edges of the type $e_{4,4}$ as shown in Figure 3 .

$$
S K_{3}(G)=\left(e_{4,4}\right)\left(\frac{4+4}{2}\right)=24
$$

\subsection{The Sanskruti index of Graphene is}

$$
S(G)=\left\{\begin{array}{lr}
389.22 t s-75.58 t-114.06 s-186.08 & \text { if } t \neq 1 \\
290.72 s-210.68 & \text { if } t=1, s>1 \\
113.76 & \text { if } t=1, s=1
\end{array}\right.
$$

Proof: Let $e_{i, j}$ denote the number of edges with $i=S u$ and $j=S v$. It is easy to see that the summation of degrees of edge endpoints of Graphene has nine edge types $e_{4,5}, e_{5,5}, e_{5,7}, e_{5,8}, e_{6,7}$, $e_{7,9,} e_{8,8}, e_{8,9}$ and $e_{9,9}$ that are enumerated Table 1 . For convenience these edge type are colored by grey, yellow, red, purple, blue, green, light blue, brown, and black, respectively, as shown in Figure 1.

Case 1. The Sanskruti index of Graphene for $t \neq 1$ is

$$
\begin{aligned}
& S(G)=\sum_{u v \in E(G)}\left(\frac{S_{G}(u) S_{G}(v)}{S_{G}(u)+S_{G}(v)-2}\right)^{3} \\
& S(G)=\left(e_{4,5}\right)\left(\frac{4 \times 5}{4+5-2}\right)^{3}+\left(e_{5,5}\right)\left(\frac{5 \times 5}{5+5-2}\right)^{3}+\left(e_{5,7}\right)\left(\frac{5 \times 7}{5+7-2}\right)^{3}+\left(e_{5,8}\right)\left(\frac{5 \times 8}{5+8-2}\right)^{3}+ \\
& \left(e_{6,7}\right)\left(\frac{6 \times 7}{6+7-2}\right)^{3}+\left(e_{7,9}\right)\left(\frac{7 \times 9}{7+9-2}\right)^{3}+\left(e_{8,8}\right)\left(\frac{8 \times 8}{8+8-2}\right)^{3}+\left(e_{9,8}\right)\left(\frac{9 \times 8}{9+8-2}\right)^{3}+\left(e_{9,9}\right) \\
& \left(\frac{9 \times 9}{9+9-2}\right)^{3} \\
& =(4)\left(\frac{20}{7}\right)^{3}+(t)\left(\frac{25}{8}\right)^{3}+(8)\left(\frac{35}{10}\right)^{3}+(2 t-4)\left(\frac{40}{11}\right)^{3}+(4 s-8)\left(\frac{42}{11}\right)^{3}+(2 s)\left(\frac{63}{14}\right)^{3}+ \\
& \quad(t-2)\left(\frac{64}{14}\right)^{3}+(2 t-4)\left(\frac{72}{15}\right)^{3}+(3 t s-4 s-4 t+5)\left(\frac{81}{16}\right)^{3} \\
& =\left(\left(\frac{25}{8}\right)^{3}+2\left(\frac{40}{11}\right)^{3}+\left(\frac{64}{14}\right)^{3}+2\left(\frac{72}{15}\right)^{3}-4\left(\frac{81}{16}\right)^{3}\right) t+\left(4\left(\frac{42}{11}\right)^{3}+2\left(\frac{63}{14}\right)^{3}-4\left(\frac{81}{16}\right)^{3}\right) s+3 t s\left(\frac{81}{16}\right)^{3}+ \\
& \quad\left(4\left(\frac{20}{7}\right)^{3}+8\left(\frac{35}{10}\right)^{3}-4\left(\frac{40}{11}\right)^{3}-8\left(\frac{42}{11}\right)^{3}-2\left(\frac{64}{14}\right)^{3}-4\left(\frac{72}{15}\right)^{3}+5\left(\frac{81}{16}\right)^{3}\right) \\
& =389.22 \mathrm{t} \mathrm{s}-75.58 \mathrm{t}-114.06 \mathrm{~s}-186.08
\end{aligned}
$$

Case 2. For $t=1$ and $s>1$, Graphene has five types of edges, namely $e_{4,4}, e_{4,5}, e_{5,7}, e_{6,7}$, and $e_{7,7}$. These edges are colored in orange, pink, red, blue, and lavender, respectively, as shown in Figure 2. The number of edges of these types is shown in Table 2. 


$$
\begin{aligned}
S(G)= & \left(e_{4,4}\right)\left(\frac{4 \times 4}{4+4-2}\right)^{3}+\left(e_{4,5}\right)\left(\frac{4 \times 5}{4+5-2}\right)^{3}+\left(e_{5,7}\right)\left(\frac{5 \times 7}{5+7-2}\right)^{3}+\left(e_{6,7}\right)\left(\frac{6 \times 7}{6+7-2}\right)^{3}+ \\
& \left(e_{7,7}\right)\left(\frac{7 \times 7}{7+7-2}\right)^{3} \\
= & (2)\left(\frac{16}{6}\right)^{3}+(4)\left(\frac{20}{7}\right)^{3}+(4)\left(\frac{35}{10}\right)^{3}+(4 s-8)\left(\frac{42}{11}\right)^{3}+(s-1)\left(\frac{49}{12}\right)^{3} \\
= & \left(4\left(\frac{42}{11}\right)^{3}+\left(\frac{49}{12}\right)^{3}\right) s+\left(2\left(\frac{16}{6}\right)^{3}+4\left(\frac{20}{7}\right)^{3}+4\left(\frac{35}{10}\right)^{3}-8\left(\frac{42}{11}\right)^{3}-1\left(\frac{49}{12}\right)^{3}\right) \\
= & 290.72 s-210.68
\end{aligned}
$$

Case 3. For $t=1$ and $s=1$, we have only 6 edges of the type $e_{4,4}$ as shown in Figure 3

$$
S(G)=\left(e_{4,4}\right)\left(\frac{4 \times 4}{4+4-2}\right)^{3}=113.76
$$

\section{Conclusion}

In this paper we have computed the Arithmetic-Geometric $\left(A G_{2}\right)$ index, $S K_{3}$ index and Sanskruti index $S(G)$ of Graphene is obtained without using computer.

\section{References}

[1] A Madanshekaf, M Moradi. The first geometric-arithmetic index of some nanostar dendrimers. Iran. J. Math. chem. 2014; 5: 1-6.

[2] D Vukicevic, B Furtula. Topological index based on the ratios of geometrical and arithmetical mean of end-vertex degrees of edges. J. Math. Chem. 2009; 26: 1369-1376.

[3] F Harary. Graph theory. Addison-Wesely. Reading mass. 1969.

[4] G Sridhar, MR Rajesh Kanna, RS Indumathi. Computation of Topological Indices of Graphene. Hindawi Publishing Corporation Journal of Nanomaterials. 2015; 1-8.

[5] Gutman. Degree-based topological indices. Croat. Chem. Acta. 2013; 86: 251-361.

[6] K Lavanya Lakshmi. A highly correlated topological index for polyacenes. Journal of Experimental Sciences. 2012; 3(4): 18-21.

[7] MV Diudea, I Gutman, J Lorentz. Molecular Topology. Romania: Babes-Bolyai University. 2001.

[8] N Trinajstic. Chemical Graph theory. Boca Raton: CRC Press. 1992.

[9] SM Hosamani. Computing Sanskruti index of certain nanostructures. J. Appl. Math. Comput. 2016; 19.

[10] SM Hosamani, I Gutman. Zagreb indices of transformation graphs and total transformation graphs. Appl.Math.Comput. 2014; 247: 1156-1160.

[11] VS Shegehalli, R Kanabur. Arithmetic-Geometric indices of some class of Graph. Journal of Computer and Mathematical sciences. 2015; 6(4): 194-199.

[12] VS Shegehalli, R Kanabur. New Version of Degree-Based Topological Indices of Certain nanotube. Journal of Mathematical Nano science. 2016; 6(1-2): 29-39.

[13] VS Shegehalli, R Kanabur. Computing Degree-Based Topological Indices of Polyhex Nanotubes. Journal of Mathematical Nano science. 2016; 6(1-2): 59-68.

[14] VS Shegehalli, R Kanabur. Computation of New Degree-Based Topological Indices of Graphene. Journal of Mathematics. 2016; 1-6. 